\title{
Nematofauna associada à cultura do quiabo na região leste de Minas Gerais
}

\author{
Rosângela D’Arc de Lima Oliveira ${ }^{1}$; Marcelo Barreto da Silva ${ }^{2}$; Naylor Daniel da Costa Aguiar ${ }^{1}$; Fábio \\ LK Bérgamo ${ }^{1}$; Alexandre Sylvio Vieira da Costa²; Lusinério Prezotti ${ }^{2}$
}

${ }^{1}$ UFV, Depto. Fitopatologia, 36570-000 Viçosa-MG; ${ }^{2}$ UNIVALE, Fac. Ciências Agrárias, 35020-220 Governador Valadares-MG; E-mail: rdlima@ufv.br; mbarreto@univale.br

\section{RESUMO}

A cultura do quiabo possui importância sócio-cultural para o estado de Minas Gerais (MG) e 34,2\% do volume de quiabo comercializado na Ceasa/Contagem procede dos municípios localizados entre Caratinga e Governador Valadares. Entretanto, o quiabeiro tem a sua produção influenciada pelos danos decorrentes de infecções causadas pelos nematóides das galhas (Meloidogyne spp.). As principais espécies desse nematóide que atacam o quiabeiro já foram relatadas no Brasil, e algumas destas podem causar a morte da planta. A correta identificação da(s) espécie(s) e, ou da(s) raça(s) de Meloidogyne presente(s) nas raízes do quiabeiro é importante na escolha da medida de controle mais apropriada. Para determinar a ocorrência e distribuição de Meloidogyne spp. e outros nematóides na região leste de MG, 70 amostras de solo e raízes da cultura, provenientes de 14 localidades, foram avaliadas por características morfológicas e isoenzimáticas. Dentre as populações de Meloidogyne spp. identificadas prevaleceu $M$. incognita (fenótipos de Esterase I1 e I2), seguida de $M$. javanica (fenótipos $\mathrm{J} 2$ e J3) $e$ M. arenaria (fenótipo A2). A espécie M. mayaguensis foi confirmada pela ocorrência do fenótipo M2 para esterase, N3 para malato desidrogenase, $\mathrm{N} 2$ para superóxido dismutase e N3 para glutamato-oxaloacetato transaminase. Este é o primeiro relato da ocorrência de $M$. mayaguensis em MG. Outros nematóides detectados na rizosfera do quiabeiro foram Aphelenchus sp., Criconemella sp., Helicotylenchus spp., Pratylenchus brachyurus e P. coffeae, Rotylenchulus reniformis, Rotylenchus sp., Tylenchus sp. e Tylenchorhynchus sp.

Palavras-chave: Meloidogyne spp., Abelmoschus esculentus, fenótipo de esterase, distribuição, hospedeiros.

\begin{abstract}
The influence of parasitic nematodes on okra crop in eastern Minas Gerais State, Brazil

Okra has a cultural and social importance for the State of Minas Gerais (MG), and $34,2 \%$ of the volume marketed in the Ceasa/ Contagem, MG, come from the municipalities located near Caratinga and Governador Valadares. The okra production is influenced for the infection caused by the root-knot nematodes (Meloidogyne spp.). The main species of these nematodes attacking okra have already been reported in Brazil, and some of them can cause plant death. The correct identification of Meloidogyne species and, or races infecting okra roots is mandatory for choosing appropriate control measures. To determine the occurrence and distribution of Meloidogyne spp. and other nematodes in the area of the East of MG, 70 soil and root samples coming from 14 municipalities were appraised for morphological and isoenzymatic characteristics. Among the populations of Meloidogyne spp., M. incognita (Esterase phenotypes I1 and I2) prevailed and were followed by M. javanica (phenotypes $\mathrm{J} 2$ and $\mathrm{J} 3$ ) and $M$. arenaria (phenotypes A2). The species M. mayaguensis was confirmed by the phenotypes: esterase (M2), malate dehydrogenase (N3), superoxide dismutase (N2) and glutamate oxaloacetate transaminase (N3). This is the first report of the M. mayaguensis occurrence in MG. Other nematodes associated with the okra plants were Aphelenchus sp., Criconemella sp., Helicotylenchus spp., Pratylenchus brachyurus and P. coffeae, Rotylenchulus reniformis, Rotylenchus sp., Tylenchus sp. and Tylenchorhynchus sp.
\end{abstract}

Keywords: Meloidogyne spp., Abelmoschus esculentus, esterase phenotype, distribution, hosts.

\section{(Recebido para publicação em 07 de agosto de 2006; aceito em 11 de abril de 2007)}

$\mathrm{O}$ quiabeiro (Abelmoschus esculentus (L.) Moench) é uma hortaliça cultivada durante todo o ano em regiões quentes e possui importância cultural e social para o estado de Minas Gerais. Em 2005, foram comercializadas 10,45 mil $\mathrm{t}$ do produto na CEASA de Contagem (MG), no valor de R\$ 11,71 milhões (CEASA/MG, 2006). Deste montante, $34,2 \%$ do volume comercializado foram procedentes dos municípios localizados entre Caratinga e Governador Valadares. Dentre eles, São João do Oriente é o segundo maior fornecedor, comercializando cerca de 1,07 mil t do produto/ano. Nesta região, onde o cultivo intensivo do quia- beiro ocorreu durante décadas, tem se observado uma redução na área cultivada, principalmente devido à presença de fitonematóides. Embora não se disponha de dados que mostrem as perdas causadas por esses organismos no quiabeiro, em especial, pelos nematóides das galhas (Meloidogyne spp.), sabese que estes patógenos constituem fator limitante de produtividade na cultura. Isto se deve provavelmente a alta suscetibilidade do quiabeiro a esses nematóides (Resende, 1986), permitindo que suas populações cresçam rapidamente no solo. A ocorrência de vários ciclos de vida do nematóide durante o período vegetativo da cultura
(Ferraz, 1980) é ainda favorecida pelas condições ideais de temperatura e umidade.

Os fitonematóides do gênero Meloidogyne são os mais importantes para a cultura, pois além de poder causar uma redução significativa na produção, em alguns casos, pode acarretar o abandono da atividade produtiva (Campos, 1995). O manejo economicamente viável desses patógenos inclui o uso de resistência varietal, entretanto não se dispõe de cultivares resistentes nacionais (Resende, 1986). Assim, o emprego da rotação de culturas com o uso de plantas não hospedeiras é de grande importância, o que torna imprescindí- 
vel o conhecimento da(s) espécie(s) presente(s) na área de cultivo.

As informações sobre a ocorrência e distribuição dos nematóides das galhas em Minas Gerais são oriundas da década de 1980, e quase nenhuma referência é feita ao quiabeiro (Ferraz, 1980; Campos et al., 1987). Visando atualizar essas informações, procurou-se, neste trabalho, estudar a ocorrência e distribuição de fitonematóides em quiabeiro, cultivado nos principais municípios produtores da região leste de Minas Gerais, e identificar as populações de Meloidogyne spp. por meio da eletroforese de isoenzimas.

\section{MATERIAL E MÉTODOS}

Para a bioprospecção dos fitonematóides foram selecionados 14 municípios que mais produzem quiabo na região, entre os municípios de Governador Valadares e Caratinga. Em cada município, foram escolhidas casualmente propriedades com a presença da cultura. O número de amostras compostas foi determinado em função da área cultivada em cada município, que variou de 4 a 40 ha. Tais áreas foram representadas por 2 a 14 amostras, num total de 70 amostras (Tabela 1). Em cada local, 12 subamostras foram coletadas para preparar cada amostra composta, constituída por cerca de $500 \mathrm{~g}$ de solo e 200 g de raízes, a qual foi acondicionada em saco plástico devidamente etiquetado, e encaminhada para o Laboratório de Nematologia da UFV. As amostras de solo foram submetidas à extração pelo método de Jenkins (1964). Das raízes, fêmeas de Meloidogyne spp. (pelo menos 26 fêmeas/amostra) foram extraídas e trituradas individualmente com pistilo em tampão de Tris- $\mathrm{HCl}$ e submetidas à eletroforese em géis de poliacrilamida no sistema vertical e descontínuo, conforme Ornstein (1964) e Davis (1964). A revelação das enzimas esterase (EST), malato desidrogenase (MDH), superóxido dismutase (SOD) e de glutamato oxaloacetato transaminase (GOT) foi feita de acordo com Alfenas (2006). Em todos os géis, o extrato protéico de $M$. javanica (Treub, 1885) Chitwood, 1949 foi aplicado em duas cavidades para servir como padrão e

Tabela 1. Freqüência (\%) de fitonematóides em amostras de quiabeiro provenientes de municípios produtores da região leste de Minas Gerais (Frequency (\%) of phytonematodes in okra samples obtained in counties of eastern Minas Gerais State). Viçosa, UFV, 2006.

\begin{tabular}{lcccccccc}
\hline $\begin{array}{l}\text { Municípios } \\
\text { (n' }{ }^{\circ} \text { de amostras) }\end{array}$ & Aph $^{1}$ & Cri & Hel & Pra & Rch & Rot & Trh & Tyl \\
\hline Alpercata (8) & & 25 & 100 & 13 & 88 & & 25 & 38 \\
Capitão Andrade (2) & & & 50 & 50 & 50 & 50 & & 50 \\
Caratinga (4) & 25 & 67 & 67 & 67 & & & & \\
Engenheiro Caldas (11) & & 10 & 82 & 18 & 64 & 9 & & 18 \\
Entre Folhas (3) & & 33 & 33 & & 33 & & & 33 \\
Fernandes Tourinho (3) & & & 67 & 67 & 100 & & & \\
Governador Valadares (3) & & & 33 & 33 & 100 & 33 & & 33 \\
lapu(2) & & & 100 & & 100 & & & \\
S. João do Oriente(7) & & 50 & 100 & 100 & & & & \\
São José do Acácio (2) & & 33 & 33 & & & & & \\
Sobrália(3) & 100 & 33 & 100 & & & & & \\
Tumiritinga(3) & 20 & 40 & 60 & 40 & 40 & 20 & & 60 \\
Ubaporanga (5) & 7 & 50 & 57 & 14 & 29 & & 7 & 50 \\
Vargem Alegre (14) & 8 & 25 & 64 & 17 & 48 & 6 & 4 & 25 \\
\hline \% em 70 amostras & & & & & & & \\
\hline
\end{tabular}

${ }^{1} \mathbf{A p h}=$ Aphelenchus sp., $\mathbf{C r i}=$ Criconemella $\mathrm{sp} ., \mathbf{H e l}=$ Helicotylenchus $\mathrm{spp} ., \mathbf{P r a}=$ Pratylenchus brachyurus ou $P$. coffeae, $\mathbf{R c h}=$ Rotylenchulus reniformis, $\mathbf{R o t}=$ Rotylenchus $\mathrm{sp} ., \quad \mathbf{T r h}=$ Tylenchorhynchus sp., Tyl = Tylenchus sp. $\left({ }^{\mathbf{A}} \mathbf{A p h}=\right.$ Aphelench $u$ s sp., Cri = Criconemella sp., Hel = Helicotylenchus spp., Pra = Pratylenchus brachyurus ou P. coffeae, $\mathbf{R c h}=$ Rotylenchulus reniformis, $\mathbf{R o t}=$ Rotylenchus $\mathrm{sp} ., \mathbf{T r h}=$ Tylenchorhynchus $\mathrm{sp} ., \mathbf{T y l}=$ Tylenchus $\mathrm{sp}$.).

assim, as mobilidades relativas das bandas foram comparadas àquelas de $M$. javanica. Fêmeas dos nematóides das galhas também foram analisadas quanto à configuração perineal (Taylor \& Netscher, 1974).

\section{RESULTADOS E DISCUSSÃO}

Nas 70 amostras provenientes dos 14 municípios da região leste de Minas Gerais (Tabela 1), Helicotylenchus spp. esteve presente em todos os municípios estudados e sua ocorrência correspondeu à freqüência de 64\% (maior incidência). $\mathrm{O}$ segundo nematóide mais freqüente foi Rotylenchulus reniformis Linford and Oliveira, 1940 (48\%), enquanto Tylenchorhynchus sp. foi o de freqüência mais baixa, presente apenas em $4 \%$ das amostras, as quais foram provenientes apenas dos municípios de Alpercata e Vargem Alegre. Considerando que os níveis populacionais dos referidos nematóides foram baixos, à exceção de algumas populações de Helicotylenchus spp., nenhuma quantificação foi realizada.

A ocorrência expressiva de espécies de Helicotylenchus nos solos cultivados não é fato inédito (Ferraz et al., 1980; Campos et al., 1987; Castro et al., 2005). Apesar de sua ampla distribuição em várias culturas, pouco se conhece sobre os prejuízos que esses nematóides podem causar à atividade agrícola. Entretanto, a presença de $R$. reniformis em 10 dos municípios amostrados merece destaque devido à crescente ocorrência desta espécie em diferentes regiões e culturas do Brasil. Como exemplos citam-se o cafeeiro em municípios de São Paulo (Kubo et al., 2004), algodão e soja no cerrado/MS (Asmus et al., 2003), cana-de-açúcar em Campos dos Goytacazes/RJ (Ferraz, S., UFV, comunicação pessoal) e em Carpina e Vicência/PE (Rosa et al., 2003), inhame da costa no Recôncavo da Bahia (Garrido et al., 2004). Essa espécie tem sido reconhecida como um problema emergente no Brasil, pois apresenta uma ampla gama de hospedeiros e distribuição nas regiões tropicais. Não se sabe o quanto $R$. reniformis é prejudicial ao quiabeiro, mas com o crescente número de áreas infestadas numa região de clima quente e cultivo intensivo, as populações podem atingir rapidamente altas densidades e causar prejuízos aos produtores de quiabo. 


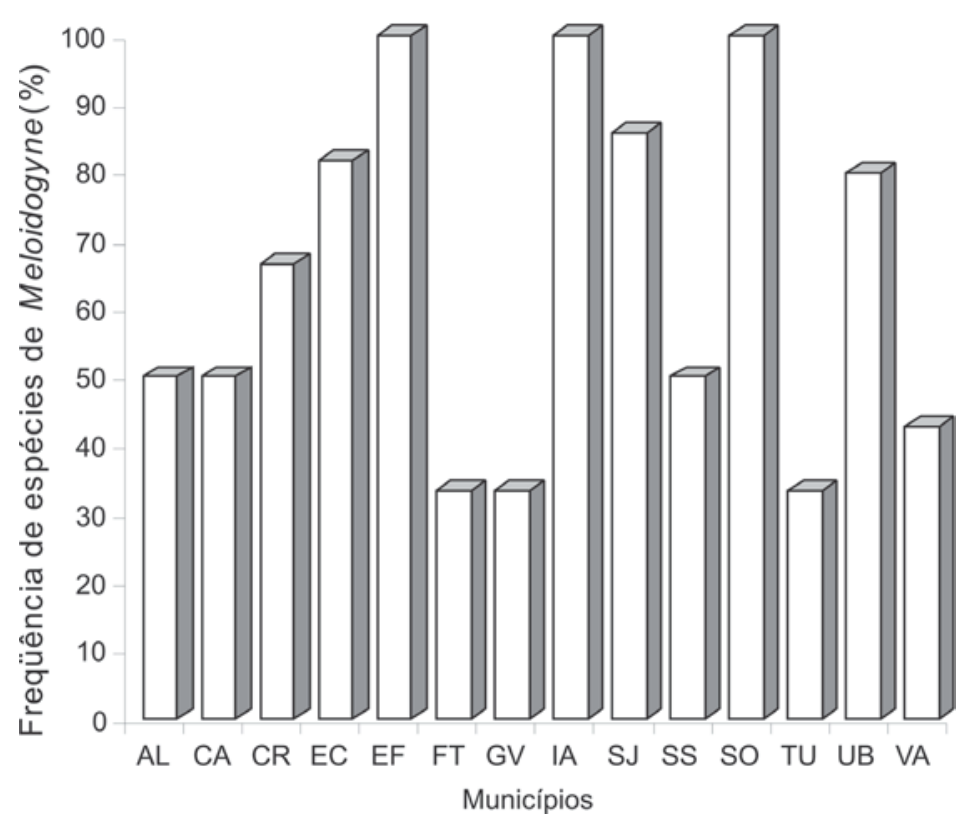

Figura 1. Frequiência (\%) de Meloidogyne spp. em setenta amostras de quiabeiro provenientes de municípios produtores da região leste de Minas Gerais. [Alpercata (AL), Capitão Andrade (CA), Caratinga (CR), Engenheiro Caldas (EC), Entre Folhas (EF), Fernandes Tourinho (FT), Governador Valadares (GV), Iapú (IA), São João do Oriente (SJ), São José do Acácio (SS), Sobrália (SO), Tumiritinga (TU), Ubaporanga (UB), Vargem Alegre (VA)] (Frequency (\%) of Meloidogyne spp. of seventy okra samples obtained in the producing counties of eastern Minas Gerais State. [Alpercata (AL), Capitão Andrade (CA), Caratinga (CR), Engenheiro Caldas (EC), Entre Folhas (EF), Fernandes Tourinho (FT), Governador Valadares (GV), Iapú (IA), São João do Oriente (SJ), São José do Acácio (SS), Sobrália (SO), Tumiritinga (TU), Ubaporanga (UB), Vargem Alegre (VA)]). Viçosa, UFV, 2006.

$\square$ Mi $\square \mathrm{Mj} \square \mathrm{Ma} \square \mathrm{Mm}$

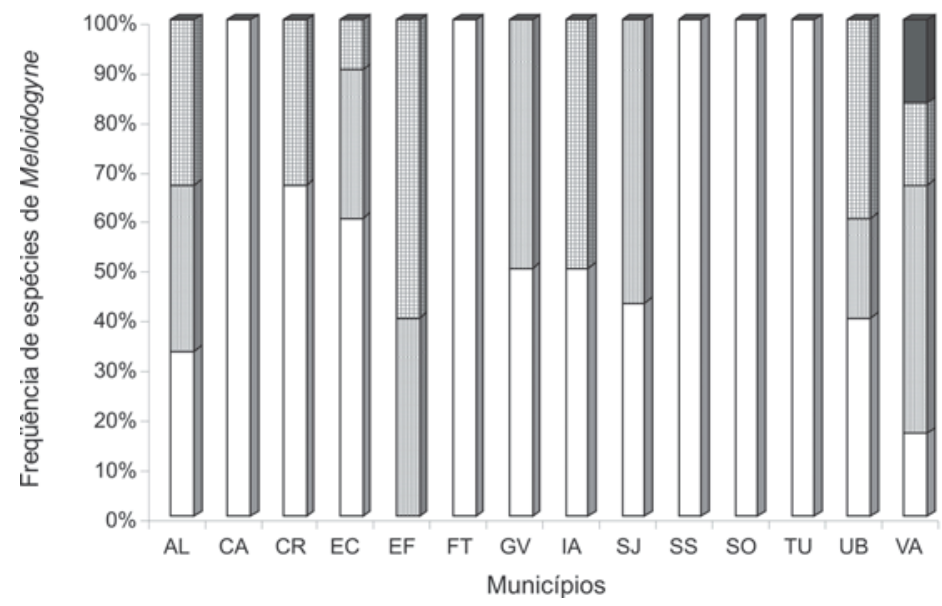

Figura 2. Frequiência de espécies de Meloidogyne em amostras de quiabeiro provenientes de municípios produtores da região leste de Minas Gerais: M. incognita (Mi), M. javanica (Mj), M. arenaria (Ma) e M. mayaguensis (Mm). [Alpercata (AL), Capitão Andrade (CA), Caratinga (CR), Engenheiro Caldas (EC), Entre Folhas (EF), Fernandes Tourinho (FT), Governador Valadares (GV), Iapú (IA), São João do Oriente (SJ), São José do Acácio (SS), Sobrália (SO), Tumiritinga (TU), Ubaporanga (UB), Vargem Alegre (VA)] (Frequency of Meloidogyne species in okra samples obtained in the producing counties of eastern Minas Gerais State: M. incognita (Mi), M. javanica (Mj), M. arenaria (Ma) e M. mayaguensis (Mm). [Alpercata (AL), Capitão Andrade (CA), Caratinga (CR), Engenheiro Caldas (EC), Entre Folhas (EF), Fernandes Tourinho (FT), Governador Valadares (GV), Iapú (IA), São João do Oriente (SJ), São José do Acácio (SS), Sobrália (SO), Tumiritinga (TU), Ubaporanga (UB), Vargem Alegre (VA)]. Viçosa, UFV, 2006.
Meloidogyne spp., comumente chamados de nematóides das galhas, foram detectados em todos os municípios numa freqüência média de $70 \%$ das amostras coletadas (Figura 1). Uma ou mais espécies estavam presentes em 100\% das amostras de Entre Folhas, Iapú e Sobrália. A menor freqüência $(33,3 \%)$ foi verificada em Fernandes Tourinho, Governador Valadares e Tumiritinga. Dentre as espécies identificadas, houve predomínio de $M$. incognita (Kofoid \& White, 1919) Chitwood, 1949 (fenótipos I1 e, raramente, I2) cuja incidência foi de $43 \%$ nas amostras coletadas (Figura 2, Tabela 2), e sua ocorrência só não foi detectada nas amostras provenientes de Entre Folhas. Em Capitão Andrade, Fernandes Tourinho, São José Acácio, Sobrália e Tumiritinga, detectou-se apenas $M$. incognita nos quiabeiros amostrados, enquanto nos demais municípios registrou-se, frequentemente, a presença de duas ou mais espécies, inclusive com a ocorrência de populações mistas numa mesma lavoura. $M$. javanica (fenótipos J2 e J3) e $M$. arenaria (Neal, 1889) Chitwood, 1949 (fenótipo A2) ocorreram em 17 e 12 amostras, respectivamente. A caracterização morfológica por meio da análise de padrões perineais das populações revelou configurações típicas de $M$. arenaria, M. incognita e $M$. javanica (Eisenback, 1985). Embora, a configuração perineal de uma das populações de Vargem Alegre lembrasse aquela de M. incognita, a identificação por meio dos padrões isoenzimáticos, principalmente de esterase (EST), revelou tratarse de M. mayaguensis Rammah \& Hirschmann, 1988, expresso por M2 (Tabela 2).

O fenótipo de EST I1 de $M$. incognita foi encontrado em todas as amostras nas quais essa espécie ocorreu, com exceção de Fernandes Tourinho, onde apenas o fenótipo I 2 foi identificado. Este fenótipo (I2) teve uma distribuição mais restrita, e foi detectado também em Alpercata e Eng $^{\circ}$ Caldas. As populações de $M$. arenaria exibiram o fenótipo A2, mas $M$. javanica foi identificada pelo fenótipo J3 em 16 das populações estudadas e pelo menos freqüente, J2, na população de São João Oriente. 
A análise pela configuração perineal resultou em padrões similares aos típicos para as espécies identificadas, isto é $M$. arenaria, M. incognita e $M$. javanica.

Os fenótipos de esterases encontrados nestas populações foram aqueles comumente encontrados para tais espécies. Uma exceção foi o fenótipo J2 de $M$. javanica, que embora já seja relatado em tomateiro e na cultura da soja (Carneiro, et al., 2000, Castro et al., 2003) é encontrado mais raramente nos solos brasileiros. Ainda que tenha havido uma correspondência entre as configurações perineais e os perfis eletroforéticos, uma das populações provenientes do município de Vargem Alegre exibiu configuração semelhante à de M. incognita, mas apresentou o fenótipo de esterase diferente daqueles I1 ou I2 exibidos por fêmeas desta espécie (Oliveira et al., 2006). O presente fenótipo, M2 (VS1-S1), foi típico de $M$. mayaguensis. Essa espécie foi relatada pela primeira vez em 2001 no Brasil, em goiabeira (PE e BA) (Carneiro et al., 2001). Posteriormente, foi também encontrado no Rio de Janeiro, Rio Grande do Norte, Ceará e Santa Catarina (Torres et al., 2004; Gomes, 2006). Esse nematóide tem sido encontrado em outros países como Cuba, Senegal, USA, França, Malawi, Trinidade-Tobago, Guadalupe, Martinica e outros, onde tem infectado várias plantas como Apium graveolens, Beta vulgaris, Brassica oleracea var. botrytis, Canavalia ensiformis, Capsicum annuum, Citrullus lanatus, Coffea arabica, Cucumis melo, Cucurbita moschata, Glycine max, Lycopersicon esculentum, Nicotiana tabacum, Phaseolus vulgaris, Petroselynum crispum, Psidium guava, Solanum tuberosum, $S$. melongena, e até na planta daninha Bidens pilosa (Brito et al., 2004; Carneiro et al., 2004, 2003, Torres et al., 2004). Brito et al. (2004) relataram também a ocorrência desta espécie em várias plantas ornamentais, inclusive em uma malvácea (Hibiscus sp.).

A ocorrência de M. mayaguensis em Vargem Alegre constitui-se no primeiro relato desta espécie no Estado de Minas Gerais. Não se sabe ainda qual o impacto da ocorrência desta espécie em Minas Gerais. A julgar pela cultura da goiaba

Tabela 2. Fenótipos de esterase (EST) detectados nas populações de Meloidogyne spp. provenientes das amostras de quiabeiros coletadas em municípios produtores da região leste de Minas Gerais (Estherase fenotypes (EST) detected on the Meloidogyne spp. populations obtained from okra samples collected in the producing counties of eastern Minas Gerais State). Viçosa, UFV, 2006.

\begin{tabular}{|c|c|c|c|c|}
\hline \multirow{2}{*}{ Municípios } & \multicolumn{4}{|c|}{ EST } \\
\hline & $\mathrm{MA}^{1}$ & M I & MJ & MM \\
\hline Alpercata & $\mathrm{A} 2$ & $|1| 2$, & J3 & \\
\hline Capitão Andrade & & 11 & & \\
\hline Caratinga & A2 & 11 & & \\
\hline Engenheiro Caldas & A2 & $|1| 2$, & J3 & \\
\hline Entre Folhas & A2 & & J3 & \\
\hline Fernandes Tourinho & & 12 & & \\
\hline Governador Valadares & & 11 & J3 & \\
\hline lapú & A2 & 11 & & \\
\hline São João do Oriente & & 11 & $\mathrm{~J} 3, \mathrm{~J} 2$ & \\
\hline São José do Acácio & & 11 & & \\
\hline Sobrália & & 11 & & \\
\hline Tumiritinga & & 11 & & \\
\hline Ubaporanga & $\mathrm{A} 2$ & 11 & J3 & \\
\hline Vargem Alegre & A2 & 11 & J3 & M2 \\
\hline
\end{tabular}

${ }^{1} \mathbf{M A}=$ Meloidogyne arenaria, $\mathbf{M I}=$ M. incognita, $\mathbf{M J}=$ M. javanica, $\mathbf{M M}=$ M. . ayaguensis

no Vale do São Francisco, pode-se esperar por perdas na produção desta olerícola e até pelo abandono das áreas cultivadas com o quiabeiro (Carneiro et al. 2001). Historicamente, essa região contribuiu grandemente para o abastecimento da Ceasa, MG, e hoje se observa uma redução de $42 \%$ na produção de quiabo no município de Alpercata, um dos maiores produtores da região há seis anos. Um dos grandes responsáveis por essas mudanças foram os nematóides das galhas (Silva MB, UNIVALE, comunicação pessoal), e com a presença de uma nova espécie na região, medidas de contenção de sua disseminação devem ser implementadas a fim de evitar maiores prejuízos aos produtores. À semelhança de outras espécies dos nematóides das galhas, sua disseminação pode ocorrer pelo uso de máquinas e implementos agrícolas na área infestada e pela água das chuvas e, ou, de irrigação. Assim, evitar que a águas de chuva e de irrigação atravessem as reboleiras infestadas e realizar a limpeza do maquinário são práticas que podem impedir que o patógeno se espalhe rapidamente.

Se a identificação desta população fosse realizada com base apenas na configuração perineal, a diagnose teria sido errônea, uma vez que o fenótipo de EST, o M2, confirmou tratar-se de $M$ mayaguensis. Desde o século passado, a configuração perineal vem sendo usada como a principal característica taxonômica na identificação de espécies de Meloidogyne, mas a tendência atual é usá-la apenas para dirimir dúvidas remanescentes de outras técnicas. O crescente número de espécies descritas no gênero e o fato da configuração perineal requerer uma análise subjetiva estimularam a busca de uma metodologia de identificação mais adequada e contribuíram para implementar o uso da eletroforese de isoenzimas. Um exemplo da subjetividade e da baixa confiabilidade desta característica é a recente descrição de $M$. paranaensis Carneiro, Carneiro, Abrantes, Santos \& Almeida, 1996. Após sua detecção em 1996, essa população foi reavaliada e descrita como uma espécie nova, com base nos estudos morfológicos e morfométricos, na resposta de hospedeiros diferenciadores e, principalmente, no fenótipo de esterase (Carneiro et al., 1996). De forma semelhante, na região do Vale do São Francisco, as primeiras populações provenientes de goiabeira a serem estudadas foram identificadas como M. incognita (Carneiro et al., 2001). Estas só foram reconhecidas como M. mayaguensis após a análise dos 
perfis isoenzimáticos. Segundo Brito et al. (2004), provavelmente as populações de M. mayaguensis já estivessem presentes na Flórida nas áreas de produção de olerícolas e ornamentais, mas somente com a utilização da eletroforese de isoenzimas na identificação de espécies de Meloidogyne é que se reconheceu a sua identidade.

Espécies novas de Meloidogyne foram descritas erroneamente no passado, por considerar que pequenas variações morfológicas, inclusive na configuração perineal, eram suficientes para caracterizar uma nova espécie. Sabe-se, atualmente, que essas variações são comumente apresentadas por esses nematóides e constituem a chamada variabilidade intraespecífica (Eisenback, 1985). Várias dessas espécies, indevidamente identificadas, foram alocadas como sinonímias de espécies bem caracterizadas, como $M$. acrita (Chitwood, 1949) Esser, Perry \& Taylor, 1976, dentre outras, que foi considerada sinonímia de $M$. incognita. Assim, ao descrever uma espécie, as características morfológicas devem ser analisadas em elevado número de espécimes, visando detectar a amplitude de variação em cada uma dessas características. Desta forma, espera-se minimizar erros quanto ao enquadramento da população em uma espécie já conhecida, ou elegêla como uma espécie nova.

A ocorrência de mais de um fenótipo para uma mesma enzima já é conhecida em outras espécies de Meloidogyne. Embora no presente trabalho só se tenha encontrado $M$. arenaria fenótipo A2, esta espécie apresenta fenótipos com 1, 2 e 3 bandas, chamadas A1, A2 e A3, respectivamente (Esbenshade \& Triantaphyllou, 1985). Enzimaticamente, $M$. incognita era identificada apenas pelo fenótipo I1 para EST, até que em 1992, um fenótipo diferente foi descrito. Esse foi chamado I 2 e apresenta duas bandas com Rm 1,00 e 1,12 (Santos \& Triantaphyllou, 1992; Carneiro et al., 1996). Contudo, ainda são escassas as informações acerca da variabilidade isoenzimática dentro das espécies de Meloidogyne, pois a eletroforese de isoenzimas ainda não é usada na rotina dos laboratórios. Com a implementação desses estudos, novos fenótipos de EST serão descobertos, e a identificação de espécies se tornará menos subjetiva e mais segura.

As populações de $M$. mayaguensis encontradas nas várias áreas de produção brasileiras geralmente exibiram o fenótipo N1a, mas a população do quiabeiro de MG mostrou o fenótipo N3 para $\mathrm{MDH}$, o que não constitui fato inédito, pois a população original deste nematóide, bem como algumas populações da Flórida, também o apresentaram (Brito et al., 2004). M. mayaguensis de MG apresentou o fenótipo N3 para a enzima GOT e o N2 para SOD, também encontrados nas populações estudadas por Carneiro et al. (2000).

Dentre os nove gêneros de fitonematóides encontrados em associação com o quiabeiro, cultivado nos 14 principais municípios produtores da região leste de Minas Gerais, Meloidogyne se destacou pela sua ampla distribuição. As espécies $M$. arenaria (fenótipo A2 para EST), M. incognita (fenótipos I1 e I2) e $M$. javanica (fenótipos J2 e J3) prevaleceram nas áreas de cultivo como espécie única ou em mistura. $M$. mayaguensis teve sua ocorrência registrada pela primeira vez em MG. Esta apresentou o fenótipo M2 para esterase, N3 para malato desidrogenase, N2 para superóxido dismutase e N3 para glutamato-oxaloacetato transaminase.

\section{AGRADECIMENTOS}

Os autores agradecem à FAPEMIG pelo financiamento do projeto.

\section{REFERÊNCIAS}

ALFENAS AC. 2006. Eletroforese e Marcadores Bioquímicos em Plantas e Microrganismos. Viçosa: Editora UFV. 627p.

ASMUS GL; RODRIGUES E; ISENBERG K. 2003. Danos em soja e algodão associados ao nematóide reniforme (Rotylenchulus reniformis) em Mato Grosso do Sul. In: CONGRESSO BRASILEIRO DE NEMATOLOGIA, 24. Anais... Petrolina: Sociedade Brasileira de Nematologia. p.169.

BRITO J; POWERS TO; MULLIN PG; INSERRA R; DICKSON DW. 2004. Morphological and molecular characterization of Meloidogyne mayaguensis isolates from Florida. Journal of Nematology 36: 232-240.

CEASA - CENTRAL DE ABASTECIMENTO DE MINAS GERAIS S/A. 2006, 23 de maio. Informações de Mercado. Em http:// w w w. c e a s a m i n a s. co m. b r / informacoes_mercado.asp/
CAMPOS VP. 1995. Doenças causadas por nematóides em alcachofra, alface, chicória, morango e quiabo. Informe Agropecuário 17: 17-22.

CAMPOS VP; LIMA RD; ALMEIDA VF. 1987. Nematóides parasitos de grandes culturas identificados em localidades de Minas Gerais e São Paulo. Nematologia Brasileira 11: 226-232.

CARNEIRO RMDG; CARNEIRO RG; ABRANTES IMO; SANTOS MSNA; ALMEIDA MRA. 1996. Meloidogyne paranaensis $\mathrm{n}$. sp. (Nemata: Meloidogynidae), a root-knot nematode parasitizing coffee in Brazil. Journal of Nematology 28: 177-189.

CARNEIRO RMDG; ALMEIDA MRA; QUÉNÉHERVÉ P. 2000. Enzyme phenotypes of Meloidogyne spp. populations. Nematology 2: 645-654.

CARNEIRO RMDG; MOREIRA WA; ALMEIDA MRA; GOMES ACMM. 2001. Primeiro registro de Meloidogyne mayaguensis em goiabeira no Brasil. Nematologia Brasileira 25: 223-228.

CASTRO JMC; CAMPOS VP; POZZA EA; NAVES RL; ANDRADE JUNIOR VC; DUTRA MR; COIMBRA JL; MAXIMINIANO C; SILVA JRC. 2005. Levantamento de fitonematóides em cafezais do Sul de Minas Gerais. In: CONGRESSO BRASILEIRO de NEMATOLOGIA, 25. Anais... Piracicaba: Sociedade Brasileira de Nematologia. p. 64-64.

CASTRO JMC; LIMA RD; CARNEIRO RMDG. 2003. Variabilidade isoenzimática de populações de Meloidogyne spp. provenientes de regiões brasileiras produtoras de soja. Nematologia Brasileira 27: 1-12.

DAVIS BJ. 1964. Disk electrophoresis. II. Method and Application to human serum proteins. Annals of the New York Academy of Sciences 121: 404-427.

EISENBACK JD. 1985. Diagnostic characters useful in the identification of the four most common species of root-knot nematodes (Meloidogyne spp.). In: SASSER JN; CARTER CC (Eds.). An advanced treatise on Meloidogyne. North Carolina: North Carolina State University. Vol. 1. p. 95-112.

ESBENSHADE PR; TRIANTAPHYLLOU AC. 1985. Identification of major Meloidogyne species employing enzyme phenotypes as differentiating characters. In: SASSER JN; CARTER CC. (Eds.). An advanced treatise on Meloidogyne. North Carolina: North Carolina State University. Vol.1. p.135-140.

FERRAZ S. 1980. Reconhecimento das espécies de fitonematóides presentes nos solos do Estado de Minas Gerais. Experientiae 26: 255-328.

GARRIDO MS; COIMBRA JL; SOARES ACF; ALMEIDA NS; PEREZ JO. 2004. Levantamento de fitonematóides na cultura do inhame da costa (Dioscorea cayennensis) no Recôncavo da Bahia. Nematologia Brasileira 28: 219-221.

GOMES CB. 2006. Ocorrência de Meloidogyne mayaguensis em fumo (Nicotiana tabacum) no Estadode Santa Catarina. In: CONGRESSO BRASILEIRO DE NEMATOLOGIA, 26. Anais... Campos dos Goytacazes: Brasília: Sociedade Brasileira de Nematologia. p. 88. 
JENKINS WR. 1964. A rapid centrifugal-flotation technique for separating nematodes from soil. Plant Disease Reporter 48: 692.

KUBO RK; OLIVEIRA CMG ANTEDOMÊNICO SAR; MONTEIRO AR FERRAZ LCCB; INOMOTO MM. 2004. Ocorrência de nematóides do gênero Pratylenchus em cafezais do Estado de São Paulo. Nematologia Brasileira 28: 159-165.

OLIVEIRADS; OLIVEIRA RDL; GONÇALVES W. 2006. Fenótipo S1 de Esterase em Meloidogyne incognita no Brasil. Fitopatologia Brasileira 31: 207-207.
ORNSTEIN L. 1964. Disk electrophoresis. I. Background and theory. Annals of the New York Academy of Sciences 121: 321-349.

RESENDE IC. 1986. Reação varietal do quiabeiro a Meloidogyne spp. e avaliação do controle por rotação com mucuna e tratamento químico de sementes. Viçosa: Universidade Federal de Viçosa. 45p (Tese mestrado).

ROSA RCT; MOURA RM; PEDROSA EMR; CHAVES A. 2003. Ocorrência de Rotylenchulus reniformis em cana de açúcar no Brasil. Nematologia Brasileira 27: 93-95.
SANTOS JM; TRIANTAPHYLLOU HH. 1992. Determinação dos fenótipos isoenzimáticos e estudos comparativos da morfologia de 88 populações de Meloidogyne spp., parasitas do cafeeiro. Nematologia Brasileira 16: 88. (Abstr.).

TAYLOR DP; NETSCHER C. 1974. An improved technique for preparing perineal patterns of Meloidogyne spp. Nematologica 20: 268-269.

TORRES GRC; COVELLO VN; SALES JUNIOR R; PEDROSA EMR; MOURA RM. 2004. Meloidogyne mayaguensis on Psidium guajava in Rio Grande do Norte. Fitopatologia Brasileira 29: 570 . 